\title{
Zur Auslese des Krankenmateriales in den Lungenheilstätten.
}

\author{
Von \\ Dr. Julian Marcuse (Mannheim).
}

Auf dem Boden der Arbeiterversicherungsgesetzgebung Deutschlands, insbesondere des Invalidenversicherungsgesetzes ist die Kreierung der Heilstätten erfolgt, in engstem Zusammenhange mit dieser ihre Existenz begründet worden. Dies schuf auf der einen Seite eine Expansion ihrer Wirksamkeit, wie sie ohnegleichen dasteht und dem Deutschen Reiche in diesem Modus der Tuberkulosebekämpfung eine Suprematie über alle anderen Kulturstaaten verliehen hat, auf der anderen aber auch gleichzeitig Gesichtspunkte fiskalischer Natur, die hemmend auf das Endziel aller dieser und ähnlicher Bestrebungen, nämlich der planmässigen Bekämpfung der Tuberkulose als Volkskrankheit im weitesten Sinne des Wortes genommen, einzuwirken geeignet erscheinen. Der klinische Begriff der Heilung wird im wirtschaftspolitischen Sinne durch den der Arbeits- bezw. Erwerbsfähigkeit ersetzt, derselbe gilt als omnipotent und bestimmt das Tun und Handeln der in Frage kommenden Instanzen, der Landesversicherungsanstalten. $\mathrm{Ob}$ als Folge der Erkrankung langdauernde Erwerbsunfähigkeit zu erwarten, inwieweit durch ein Heilrerfahren dauernde und erhebliche Erwerbsfähigkeit hergestellt werden könne, dies sind die leitenden Gesichtspunkte für die Versicherungsanstalten, dies die Maximen ihres Vorgehens. Und innerhalb dessen ist der weiteste Spielraum den jeweiligen Anschauungen gelassen, ob und bis zu welchem Grade die Zugehörigkeit zur Invaliditätsversicherung die Befugnis zur Übernahme eines Heilverfahrens gewährleistet, mit anderen Worten, welche Dauer geleisteter Arbeit als notwendige Voraussetzung der reichsgesetzlichen Fürsorge anzusehen ist. Diese 
weitgehende diskretionäre Vollmacht in einer die Vitalität der Bevölkerung aufs tiefste beeinflussenden Frage rührt eben von der Unterordnung der Heilstättenbehandlung der Lungentuberkulose unter die Bestimmungen des Invalitätsversicherungsgesetzes her, deren für die vorliegende Frage massgebender $\$ 18$ bekanntlich lautet: Ist ein Versicherter dergestalt erkrankt, dass als Folge der Krankheit Erwerbsunfähigkeit zu besorgen ist, welche einen Anspruch auf reichsgesetzliche Invalidenrente begründet, so ist die Versicherungsanstalt befugt, zur Abwendung dieses Nachteils ein Heilverfahren in dem ihr geeignet erscheinenden Umfang eintreten zu lassen. Diese in dem Rahmen eines sozialpolitischen Gesetze sich bewegenden hygienischen Aufgaben müssen durch dasselbe bestimmte Einengungen erfahren, die eine Gefährdung des fundamentalen Prinzipes aller Bestrebungen zur Hebung der Volksgesundheit in sich schliessen, nämlich der uneingeschränkten, ausnahmslosen Berücksichtigung sämtlicher von einer bestimmten Krankheit befallener Individuen und ihres vollen Anspruches auf Besserung und Heilung, soweit dies überhaupt möglich ist. Diese Einengungen werden diktiert durch die grundlegenden Bestimmungen des Gesetzes, wonach mindestens einhundert geleistete Beiträge als Norm für die Gewährung einer Rente anzusehen sind, und sie haben zur Folge einmal den Nachweis einer bestimmten Zahl geklebter Marken für die eventuelle Einleitung eines Heilverfahrens und weiterhin eine rigorose Art der Krankenauslese, die scharfe Betonung des Frühstadiums der Erkrankung. Der erstere Gesichtspunkt unterliegt der mehr oder minder humanen Auslegung seitens der Ausschüsse der Versicherungsanstalten und zeitigt demzufolge die heterogensten Auffassungen: Während von den einen die Beibringung von fünfzig Marken verlangt, ja sogar in glücklicherweise vereinzelten Fällen ein Anspruch auf Invaliditätsrente erst als massgebend für Einleitung eines Heilverfahrens angesehen wird, gibt es Versicherungsanstalten, die sich mit dreissig Marken und noch weniger begnügen. Der zweite Gesichtspunkt der scharfen Auslese ist ein von allen Anstalten gleichmässig betonter: er basiert auf der Tatsache, dass bei den eigenen Voraussetzungen, unter denen die Heilstätte arbeitet, nur bei den Anfangsstadien der Erkrankung sich die geforderte längerdauernde Erwerbsfähigkeit von mindestens $331 / 3 \%$ später nachweisen lässt. Diese Voraussetzungen sind, wie dies $\mathrm{Brauer}{ }^{1}$ ) exakt formuliert hat, die Aufgaben der Heilstätte, nämlich in einer begrenzten Zeit die Arbeitsfähigkeit ihrer Pfleglinge wieder herzustellen oder zu befestigen, die Kranken möglichst spät zu invaliden Renten-

1) Den Einfluss der Krankenversorgung auf die Bekäınpfung der Tuberkulose als Volkskrankheit. Beiträge zur Klinik der Tuberkulose, Bd. II, Heft 2. 
empfärngern werden zu lassen und alles dies gegenüber einem Menschenmaterial, das nach der Entlassung vielfach in unhygienische Verhältnisse zurückkehren muss. Diese beiden eben kurz charakterisierten Methoden gegenwärtiger Handhabung des $\S 18$ des Invaliditätsgesetzes bedeuten ein schweres Hemmnis für die Überwindung der Tuberkulose im Volkskörper, indem sie einmal Anfangsstadien der Erkrankung, die den fiskalischen Ansprüchen nicht Genüge leisten, in die Armee der vorgeschrittenen Kranken nach und nach hinabstossen, und indem sie weiterhin die letzteren, wenn abgelehnt, zur Quelle steter Infektion für ihre Umgebung sowie zu ihrer eigenen allmählichen Konsumption werden lassen. Damit begibt sich die Heilstätte des vornehmsten Prinzipes der Antituberkulosebewegung, der Verhütung stets wiederkehrender Neuerkrankungen, und ihr Nutzwert für die Bekämpfung der Lungenschwindsucht als Volkskrankheit sinkt erheblich. Inwieweit diese Einwürfe begründet sind, mögen die folgenden statistischen Zahlen lehren. Sie rühren aus dem soeben verflossenen Geschäftsjahr 1904 her und betreffen den Kreis der Versicherten der Ortskrankenkasse Mannheim I. In diesem Jaliro wurden insgesamt 242 Heilverfabren beantragt, von denen nur 127 : tsächlich zur Ausführung gelangten, während 115, das sind $90,5^{\circ} ; 0$ ron der Versicherungsanstalt Baden bezw. in zwei Fällen von der Versicherungsanstalt der Pfalz abgelehnt wurden. Diese 115 Fälle verteilen sich in ihren ursächlichen Momenten auf folgende Kategorien: Wegen mangelhaften Markenklebens bezw. Aufhebung des Versicherungsverhältnisses wurden abgelehnt 13, wegen disziplinärer Vergehen 8 , bei weiteren 9 trat während der Wartezeit bis zur definitiven Einberufung eine so erhebliche Besserung ein, dass vorderhand ein Heilverfahren nicht mehr indiziert war, 4 entzogen sich jedweder Kontrolle bezw. waren infolge Verschlimmerung des Zustandes nicht mehr transportabel. Die Mehrzahl der wegen ungenügender Beitragsleistung Zurückgewiesenen waren Anfangsstadien der Erkrankung und Träger der Infektion für ihre Umgebung, da sie in der weitaus überwiegenden Zahl verheiratet waren. Die wegen sogenannten disziplinärer Vergehen Abgelehnte waren Patienten, die bereits einmal in einer Lungenheilstätte gewesen waren und sich aus äusseren oder inneren Gründen - zum Teil familiäre Veranlassungen - der Behandlung bis zum Schluss entzogen hatten. Es mag dahingestellt bleiben, inwieweit Billigkeitsgründe nicht hier von Fall zu Fall hätten ausschlaggebend sein müssen, um eine Wiederholung der Kur einzuleiten, da in sämtlichen Fällen böswillige Momente nicht vorlagen. Der gesamte übrige Teil, nämlich 81, wurden aus Motiven, die mit dem Krankheitsprozess als solchem zusammenhingen, abgelehnt. 35 
davon waren bereits einem Heilverfahren unterzogen worden, und der Rückfall genügte, um die Versicherungsanstalt zu einem ablehnenden Bescheid gelangen $z \mathfrak{u}$ lassen. Denn neben einem Bruchteil wesentlicher Verschlimmerungen und Übergreifen des Prozesses auf bis dahin intakte Lungenpartien stehen eine Reihe von Kranken, bei denen nur eine Wiederholung des ursprünglichen lokalisierten Krankheitsstadiums zu konstatieren war. Die Nichtüberweisung in die Heilstätte ist für diese Kategorie wohl allein aus gewissen fiskalischen Erwägungen heraus zu erklären, es besteht eben bei den Versicherungsanstalten, da wo nur die Möglichkeit nicht aber die Wahrscheinlichkeit einer Wiedererlangung der Erwerbsfähigkeit vorliegt, keine Neigung, dazu die Hand zu bieten. Das mag im Interesse der Rentabilität der Heilstätten diskutabel sein, die Bekämpfung der Tuberkulose als Volkskrankheit kann aber bei diesen Maximen nie gefördert werden, und die Versicherungsanstalten begeben sich ihres Anspruches als Träger der Antituberkulosebewegung. Ihre rigorose Praxis äussert sich aber nicht bloss nach unten, da wo vorgeschrittene Krankheitsstadien bereits einem Heilverfahren Einhalt bieten, sondern auch nach oben, wo der fehlende oder unsichere objektive Befund die tuberkulöse Natur des Leidens nicht völlig einwandfrei erkennen lässt. In dem mir vorliegenden Material registriere ich 23 solcher Fälle, bei denen die von den behandelten Ärzten konstatierten krankhaften Veränderungen der Lungen als nicht ausreichend zur Einleitung eines Heilverfahrens seitens der Versicherungsanstalten angesehen wurden. Die hieraus entstehenden Folgen involvieren nicht nur die Herabsetzung der Widerstandsfähigkeit des Einzelindividuums, sondern machen auch die allgemeinen sozialhygienischen Massnahmen zur Eindämmung der Tuberkulose illusorisch. Und welche Widersprüche im konkreten Fall sich ergeben, dies möge aus folgenden drei Krankengeschichten erhellen: L. Sch., 18 Jahre alt, wird wegen beginnender Tuberkulose vom behandelnden Arzt zur Einleitung eines Heilverfahrens eingegeben, die Vorbeobachtungsstation der Versicherungsanstalt sieht den erhobenen Befund als nicht ausreichend an, lehnt die Übernahme der Patientin ab und verweist sie in ein Erholungsheim. Dort wird nach mehrmonatlichem Aufenthalt keine Besserung erzielt, der beaufsichtigende Arzt konstatiert ebenfalls Initialstadium der Lungenschwindsucht, und jetzt erst entschliesst sich die Versicherungsanstalt zur Überführung der Patientin in eine Lungenheilanstalt. In zwei weiteren Fällen führte die beteiligte Ortskrankenkasse nach Ablehnung seitens der Versicherungsanstalt wegen Aussichtslosigkeit der Wiederherstellung der Erwerbsfähigkeit ein Heilverfahren auf Kosten der Kasse durch und erreichte beide Male einen vollen Erfolg, das heisst wesent- 
liche Besserung des Zustandes und bis zum Augenblick andauernde Arbeitsfähigkeit.

Aus alledem ist ersichtlich, dass fiskalische Gesichtspunkte in erster und bedeutsamster Weise massgebend sind für die Praxis der Versicherungsanstalten, und dass von diesen Gesichtspunkten auch die von den Anstalten ins Leben gerufenen Vorbeobachtungstationen, wie natürlich, geleitet werden. Nur die Frage, inwieweit ein Heilverfahren den Rentenbezug aufschieben bezw. es verhindern kann, dass die Kranken zu invaliden Rentenempfängern werden, ist ausschlaggebend für die Massnahmen der Versicherungsanstalten. Daher die strenge Betonung der Frühdiagnose, daher die strenge Auslese sowie die fast ausnahmslose Ablehnung aller derer, die nach relativ kurzer Zeit, nachdem sie entlassen waren, erneut erkranken. Im Rahmen der sozialpolitischen Gesetzgebung mag daher die Heilstätte ihre Aufgabe erfüllen, den grossen Prinzipien der Antituberkulosebewegung aber dient sie nur in unvollkommenstem Masse. Hier werden, was schon Brauer energisch betont hat, eine Reihe von Massnahmen zu treffen sein, die einmal von rein ärztlichen und klinischen Gesichtspunkten ausgehen und die Krankheit als solche zum alleinigen Zielpunkt ihres Vorgehens nehmen und die weiterhin im sozialhygienischen Interesse der Frage nachzugehen suchen, wie die Infektionsgefahr für die Gesunden zu vermeiden ist. Diesen Zwecken dienen im Anschluss an die Krankenhäuser und Kliniken zu errichtende Tuberkulosestationen und ferner Heimstätten für die Schwerkranken bezw. für unheilbare Tuberkulöse. Erst mit ihrer beider Inaugurierung werden Individualhilfe wie Volksschutz - diese beiden Endzwecke einer rationellen Seuchenbekämpfung - im Kampfe gegen die Tuberkulose erreicht werden können. 